\title{
Cognitive Tasks in Intelligence Analysis: use of Event Dwell Time to Characterize Component Activities
}

Thomas F. Sanquist, Frank L. Greitzer, Antoinette Slavich, Rik Littlefield, Janis Littlefield, and Paula Cowley

For Presentation at:

Human Factors and Ergonomics Society $48^{\text {th }}$ Annual Meeting

September 20-24, 2004

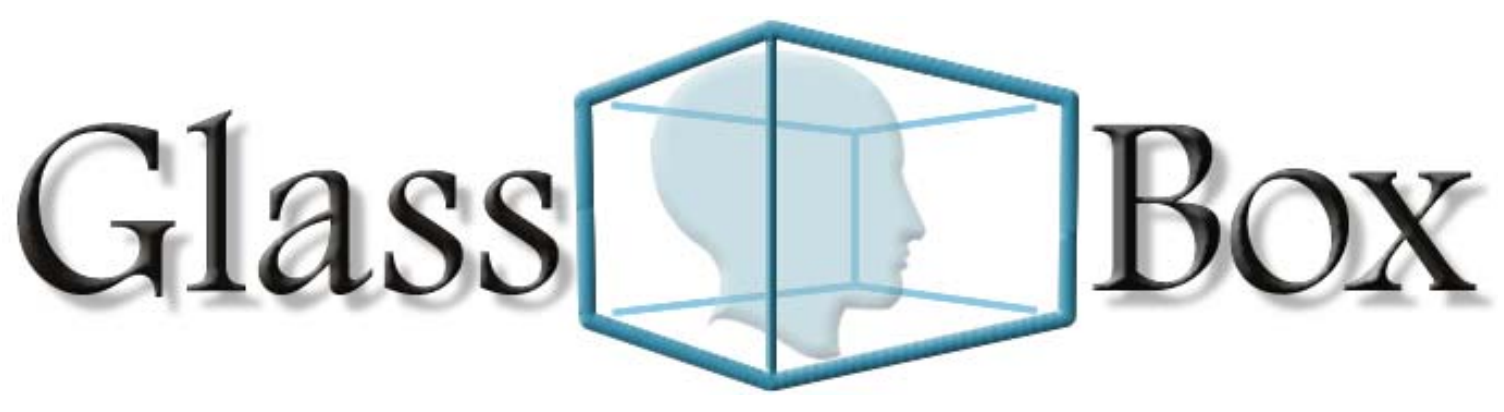

Battelle Memorial Institute 


\title{
Cognitive Tasks in Intelligence Analysis: Use of Event Dwell Time to Characterize Component Activities
}

\author{
Abstract \\ Thomas F. Sanquist, Frank L. Greitzer, Antoinette Slavich, \\ Rik Littlefield, Janis Littlefield, and Paula Cowley \\ Battelle Pacific Northwest Division
}

Technology-based enhancement of intelligence analysis requires a detailed understanding of the cognitive tasks involved in the process. The information search and report production tasks of the intelligence analysis process were investigated through evaluation of time-stamped workstation data gathered with custom software. Model tasks simulated the search and production activities, and a sample of actual intelligence analyst data were also evaluated. Task event durations were calculated on the basis of millisecond-level time stamps, and distributions were plotted for analysis. The data indicate that task event time shows a cyclic pattern of variation, with shorter event durations ( $<2 \mathrm{sec}$ ) reflecting information search and filtering, and longer event durations (> $10 \mathrm{sec}$ ) reflecting information evaluation. Application of cognitive principles to the interpretation of task event time data provides a basis for developing "cognitive signatures" of complex activities, and can facilitate the development of technology aids for information intensive tasks. 


\title{
Summary
}

\section{Cognitive Tasks in Intelligence Analysis: Use of Event Dwell Time to Characterize Component Activities}

\author{
Thomas F. Sanquist, Frank L. Greitzer, Antoinette Slavich, \\ Rik Littlefield, Janis Littlefield, and Paula Cowley \\ Battelle Pacific Northwest Division
}

\section{$\underline{\text { Introduction }}$}

Homeland security depends to a great extent on human intelligence analysts who gather and process large volumes of information to develop products reflecting the capabilities and intentions of potential adversaries. This inherently cognitive activity has been the subject of process-level research (Patterson, et al., 2001), but is difficult to study at the component level because of the unconstrained nature of the work. This report demonstrates an approach to collecting and analyzing workstation data from working intelligence analysts, with a focus on the use of temporal measures to describe their task-level activities. We anticipate that the focus on time will allow us to characterize those cognitive activities that are amenable to augmentation with technology.

Psychologists have long employed time as a measure for evaluating the linkage between external behavior and cognitive processes (Meyer, et al., 1988). Although most of this work tends to focus on the millisecond time domain, the same principles can be applied to behavior observed in non-experimental situations. These include:

- Duration of behavior reflects duration of component cognitive processes.

- Repetitive or "automatic” processes take less time.

- Conscious (“controlled”) or more complex processes take longer.

- Temporal patterns can be used as indicators of cognitive processes.

These principles can be used to interpret the temporal aspects of intelligence analysis tasks based on behavioral data obtained in the typical search-analyze-report cycle.

\section{Method}

The research approach involves a combination of model task analysis, and case study of a single subject (Franklin, Allison and Gorman, 1997). The enabling technology is a suite of custom software referred to as the Glass Box Analysis Environment (GBAE). In essence, this software captures and time stamps all events that occur on a Windows workstation - individual keystrokes, window launch and sizing, and numerous other operator and system-based actions. The primary function of the GBAE software is to collect workstation data while intelligence analysts perform open-source information searches and report production.

We recognize that there is more to intelligence analysis than information search and report production; activities such as collaboration, telephoning, use of hard copy and off-site resources 
are all routine components. However, because of the increasingly heavy reliance intelligence analysts place upon Internet resources, we have chosen to focus our efforts in this arena.

The GBAE collects and time stamps workstation data at a very high rate. In order to develop an approach to reducing this volume and discerning high-information yield behaviors in the data, we initially carried out three model tasks. The model tasks were executed by a senior researcher with extensive experience in conducting information searches via the Internet.

The first model task involved conducting an open source Web-based search for items of interest in the area of Psychological Operations in information warfare. The task was conducted using the Google search engine and Internet Explorer as a browser. The search task lasted 40 minutes and yielded 308 discrete Glass Box events. In order to better understand the relationship between events that comprise "search result filtering" versus "reviewing items of interest," the second model task involved seeking a single item of interest on the general topic of "central nervous system effects of biological weapons." The total session time for this model task was 7.6 minutes, yielding a total of 93 Glass Box data events. To evaluate dwell time patterns in a somewhat different type of task, the analyst used the Web pages of interest that were found in Model Task 1 as the basis for a $3 / 4$ page summary of the findings. The total session time for this model task was 18 minutes and generated 136 Glass Box data events. To determine the extent to which similar patterns might be observed in actual intelligence analyst activity, we selected a segment of data from a single government-contracted intelligence analyst who has been conducting his work in the GBAE for the past six months. This session sample was 48 minutes in duration and generated 437 events.

\section{Time Measure Computation}

GBAE data were filtered to eliminate system-generated events, and arrayed in an Excel spreadsheet for analysis. The time stamps were used to calculate event dwell time as:

$$
\text { Event Dwell Time }(\text { Row } N)=\text { Time in Row }(N+1)-\text { Time in Row } N \text {. }
$$

This metric can be used to evaluate the relationship between events according to their duration, patterns of event duration, and relationships between application usage and time.

\section{$\underline{\text { Results }}$}

The principal data analysis methods for this task analytic and case study approach involved visual inspection of dwell time distributions, analysis of time interval frequency data, and analysis of temporal patterns in application usage. The SPSS software package was used to construct the plots and time interval distributions.

Figures 1 - 4 show the dwell time data across events arranged chronologically. The results depicted in Figure 1 show a cyclic pattern of alternation between events that have durations of less than 10 seconds, and those that exceed 10 seconds. Figure 2 shows the dwell time data for the single item search. This plot also shows the preponderance of data events at the sub-10 second duration, with cyclic activities at the 10-35 second duration, and clear peaks at events 40 and 43. Evaluation of the specific event data indicates that the user was reading on-screen Web pages related to the target search topic. The remaining events are associated with launching 
Word, establishing storage folders for the search, and various cut/paste operations to save the page.

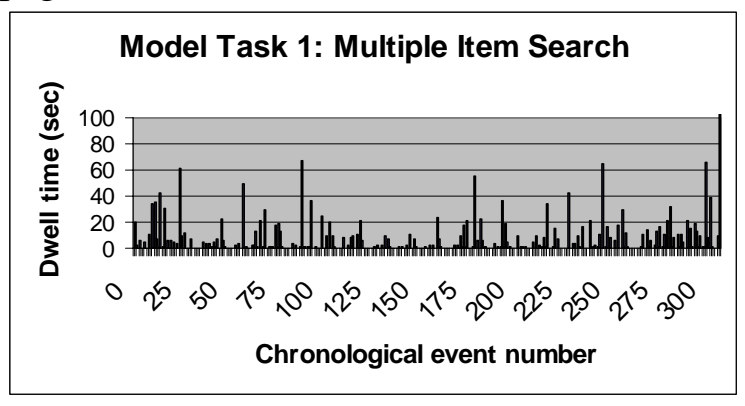

Figure 1. Dwell Time for Multiple Item Search Task. Session duration $=40 \mathrm{~min}$.

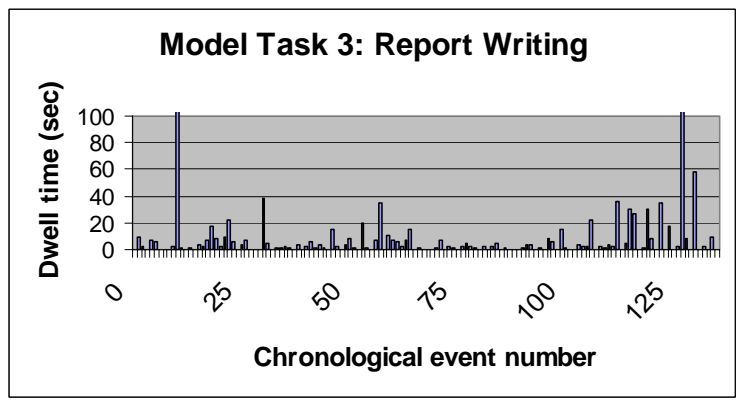

Figure 3. Dwell Time for Report Writing Task. Session duration $=18 \mathrm{~min}$.

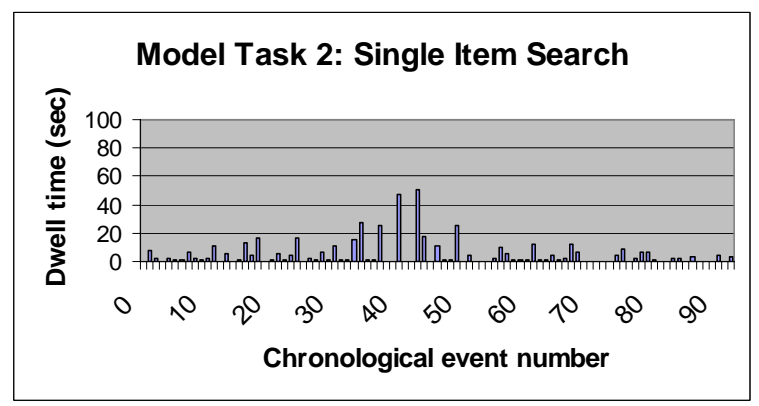

Figure 2. Dwell Time for Single Item Search Task. Session duration $=7.6 \mathrm{~min}$.

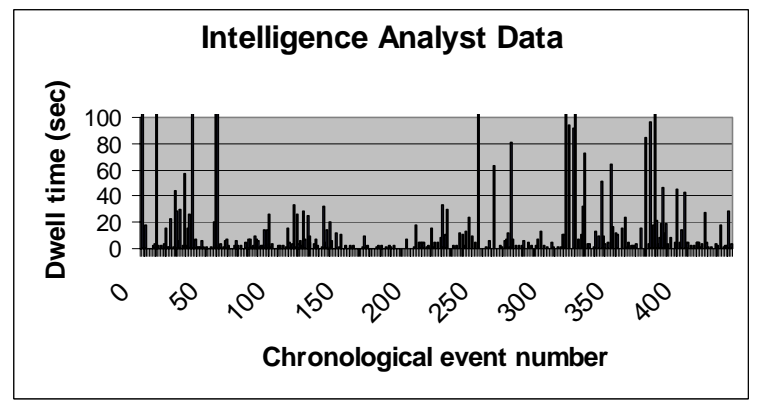

Figure 4. Dwell Time for a Sample Glass Box Analysis task. Session duration $=48 \mathrm{~min}$.

Figure 3 illustrates the results of the report production model task. The plot has a similar structure to Figures 2 and 3, with an apparent cyclic activity that manifests in several long duration peaks, with some other cyclic activity appearing in the $10-35$ second duration region. Evaluation of the corresponding event data indicates that the peaks between events 0 and 25 and between events 125 and 136 are associated with 2.5 minutes and 4 minutes respectively in Word, in which text was being typed into a document. Peaks in the $10-35$ second region represent access to stored Web pages that the analyst was consulting for material relevant to his written report.

In Figure 4, it is clear that there is a peak of 100 seconds or greater around event 50 . The specific event number is 55, which showed a dwell time of 625 seconds, or more than 10 minutes. The preceding events indicate that the analyst opened a Word file. The keyboard and mouse activity associated with this 625-second event shows several mouse clicks followed by 78 "page down" key presses. This indicates that the analyst was reading the document on the screen. These data from an actual analyst performing real work reinforce the view that dwell time measures can be used to gain insight regarding cognitive activity during a session. Data such as these can be used to identify reading, which is accompanied by keyboard data - in this case, a key press about every 8 seconds. If a similarly long period of time occurred without keyboard or mouse activity, it would indicate the analyst's attention is elsewhere.

The data illustrate that for all four tasks, roughly half of the total events have dwell times of 2 seconds or less. This suggests that most of the discrete events consist of rapid viewing of material, discarding it, and moving on to another display. Thus, most of the activity represented would fall into either automated pre-perceptual processes or rapid scanning and filtering. 
Elapsed time per application shows the overall usage level of various software applications. However, information concerning the temporal pattern of usage is also informative, because it conveys how the user switches between activities over time, and can be useful for understanding the flow of information across activities. Figures 5 - 8 illustrate the temporal pattern of application usage in Model Tasks 1 - 3, and the Intelligence analyst data.

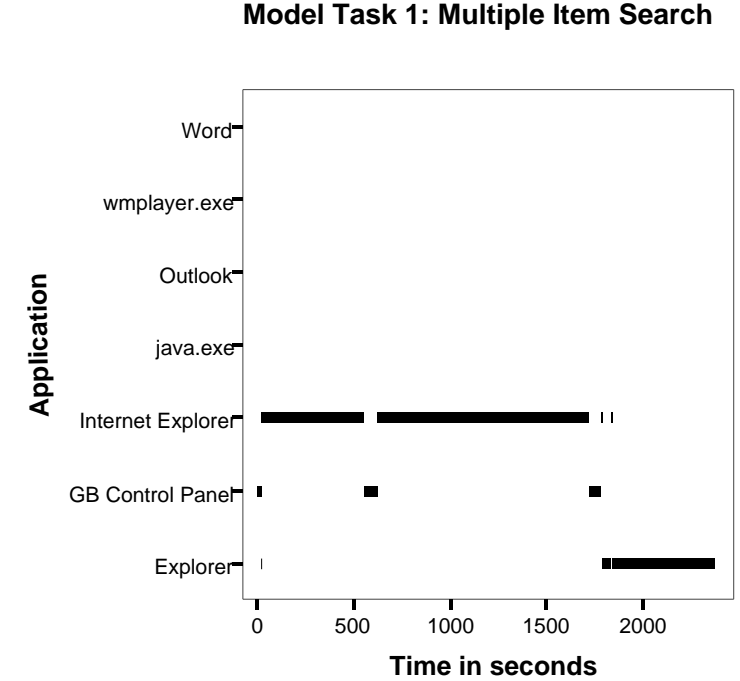

Figure 5. Timeline Line Plots of Application Utilization in Model Task 1.

Model Task 3: Report Writing

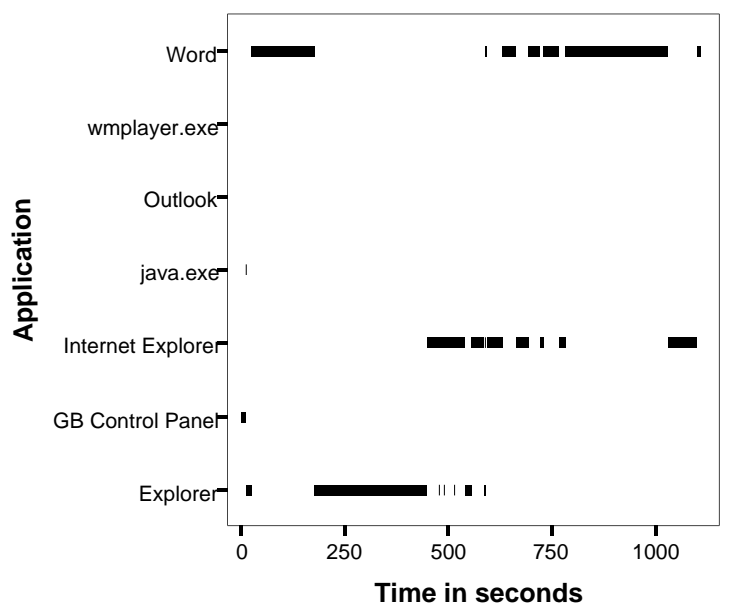

Figure 7. Timeline Plots of Application Utilization in Model Task 3.

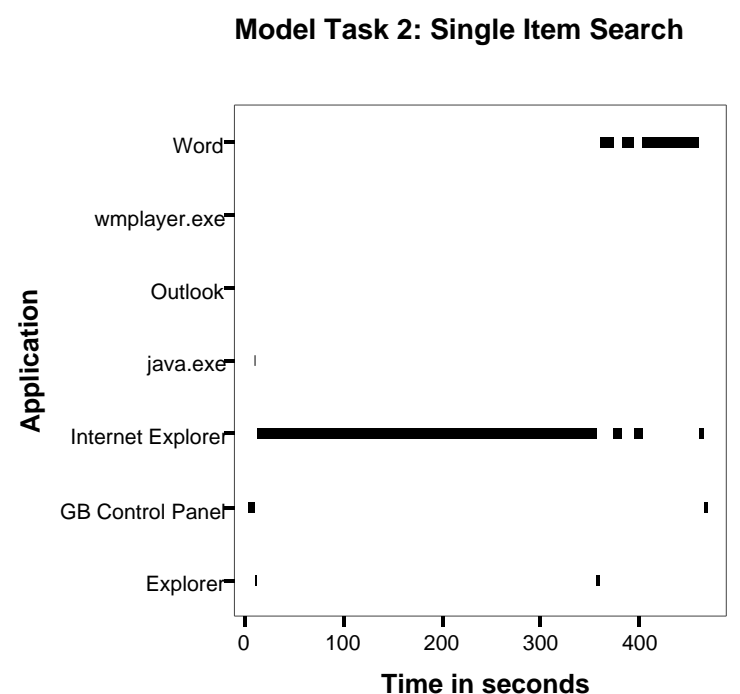

Figure 6. Timeline Plots of Application Utilization in Model Task 2.

Intelligence Analyst Data

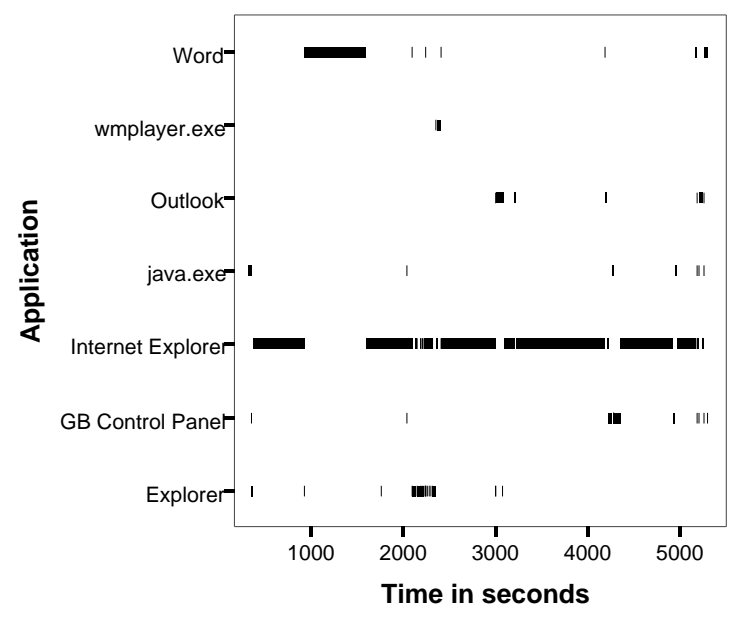

Figure 8. Timeline plots of Application Utilization in Glass Box Analyst task.

\section{Discussion and Conclusions: Prospects for Application of Time Measures}

This paper has illustrated several approaches to analysis of time data that can be derived from the GBAE data. The analyses suggest that time measures provide a useful way of (1) initially screening data to characterize the nature of the analyst session, (2) identifying specific areas 
where the analyst shows interest, (3) identifying the material that is considered "noise" by the analyst (material filtered out with quick views), and (4) portraying the flow of information across the task structure. These elements provide a set of converging measures to interpret the unobservable cognitive processes comprising intelligence analysis.

Analysis of dwell time interval distributions may prove useful in identifying cycles of scanning/filtering and focused attention that would be associated with alternating patterns of shorter and longer dwell times. This type of cycle corresponds to a common experience while using the Internet for information search: rapid perusal of mostly irrelevant information, followed by somewhat more interesting (or at least difficult to decide about) information, and finally a "hit" that leads to focused attention and reading.

There is also some indication that just prior to a focus of interest as shown by a long dwell time, there is an increase in event durations. This type of finding, if confirmed, would indicate that users have caught the "scent" for the information they seek. Machine-aided focusing in these "likely areas" would facilitate more time spent evaluating and analyzing, and less time spent trying to get to the right place. Additional GBAE data are currently being collected from working intelligence analysts; future activities include describing the entire analytic cycle, and development of "cognitive signatures" to facilitate machine-aided job performance. Such enhancements of the intelligence analysis process will contribute to increasing the efficiency and precision of this important homeland security activity.

\section{$\underline{\text { References }}$}

Franklin, R.D., Allison, D.B. and Gorman, B.S. (Eds.). (1997). Design and Analysis of SingleCase Research. Mahwa, NJ: Lawrence Earlbaum Associates.

Meyer, D.E., Osman, A.M., Irwin, D.E., and Yantis, S. (1988). Modern mental chronometry. Biological Psychology. 26: 3 - 67.

Patterson, E.S., Woods, D.D., Roth, E.M., Finley, J.M., and Kuperman, G.G. (2001). Aiding the Intelligence Analyst in Situations of Data Overload: From Problem Definition to Design Concept Exploration. Institute for Ergonomics/Cognitive Systems Engineering Laboratory Report, ERGO-CSEL-01-TR-01. 